\title{
NUMERICAL STUDY USING CFD METHODS OF THE SHAPES OF A SPORT BOAT WITH THE HULL MADE OF ALUMINUM
}

\author{
Costică Hogaș \\ "Dunarea de Jos" University of Galati, \\ Faculty of Naval Architecture, Galati, Domneasca \\ Street, No. 47, 800008, Romania, \\ E-mail: costica.hogas@glo-marine.com

\section{Costel Iulian Mocanu} \\ "Dunarea de Jos" University of Galati, \\ Faculty of Naval Architecture, Galati, Domneasca \\ Street, No. 47, 800008, Romania, \\ E-mail:costel.mocanu@ugal.ro
}

\author{
Liviu Andrei Moise \\ "Dunarea de Jos" University of Galati, \\ Faculty of Naval Architecture, Galati, Domneasca \\ Street, No. 47, 800008, Romania, \\ E-mail: liviu.moise@glo-marine.com

\section{Eugen Găvan} \\ "Dunarea de Jos" University of Galati, \\ Faculty of Naval Architecture, Galati, Domneasca \\ Street, No. 47, 800008, Romania, \\ E-mail: eugen.gavan@ugal.ro
}

\begin{abstract}
Taking into account the latest requirements for reducing the pollution that results from the combustion of fossil fuels, there was a need to reduce the power of ships propulsion engines. In this context, the requirement are also available for sport boats. So in order to reduce the power of the propulsion engine, solutions are sought to reduce the total resistance of the ship. This is done by reducing the wetted surface of the hull. In this paper are presented two concepts of sport boats, as well as the comparison between them, whose body is designed to be made of aluminum. In order to optimize the shapes using CFD methods, it was studied the flow around the ship's hull in various constructive ways.
\end{abstract}

Keywords: boat, CFD, shape, flow, wave

\section{INTRODUCTION}

Small ships, unlike big ships (the conventional ones) differ in smaller size, different destination and behavior during navigation.

The sustaining forces that appear when a ship is moving on the free surface of the water, determines its navigation regime. In the case of sport boats, sustained hydrodynamic forces increase as the speed of ship movement increases, resulting in gradual exit from the water and reduced wetted surface of the hull. The navigation regime of a boat where sustainability is determined by hydrodynamic forces is called planning regime.

The determination of the ship's displacement regime is based on the Froude number, calculated with volumetric displacement $\mathrm{Fn}_{\bar{v}}$. The Froude number is calculated with the equation [1]:

$F n_{\bar{\eta}}=\frac{v}{\sqrt{g \cdot \nabla^{1 / 2}}}$

For the ship to be in the planning regime, the condition $\mathrm{F}_{n_{\eta}}>3$ must be fulfilled. However, taking into account that the calculation time of a model in planning regime is very high, the speed will be slower so the ship does not exced the transient regime, and 
after obtaining an optimal shape of the hull it goes to the next step. That means the optimal model will be analyzed in planning regime.

In order to obtain an optimal shape that will lead to minimal drag force and efficient sliding through the water, CFD (Computational Fluid Dynamics) methods will be used to allow us to analyze the flow around the hull of the ship to see the phenomena that occur and to optimize the areas that lead to detachments.

The first analyzed shape has specific forms for these type of boats, namely a sharp bilge whith $\mathrm{V}$-shaped bottom. The angle of the bottom in transversal section increases from the stern to bow.

The shape plan was generated with the help of Tribon software, Lines module. After that based on the lines plan the surface of the hull was generated in Rhinoceros.

For the first shape, common shapes have been adopted so that on the basis of the results obtained by means of the numerical method, a gradual optimization of the shape can be made.

For the second shape was chosen the variant of a curved $\mathrm{V}$-shaped bottom and a longitudinal redan on the bilge area. Planning hull with curverd V-shaped bottom show superior hydrodynamic performances and at the same time lower hydrodynamic load on waves.

The adopted shapes take into account both fluid flow factors around the hull and the technological factors of making the body of the ship, so as to find the optimal way between prince-size and ship-propulsion power.

These criteria formed the basis of the choice of the two shapes for the hull of the ship, and by the test that will be made in the towing tank, we will decide if the second shape is the one to be adopted. It is assumed that the second shape is optimal because by the observations it is deduced that the longitudinal redan will substantially reduce the waves and implicitly the total ship resistance with the corresponding decrease of the propulsion power.

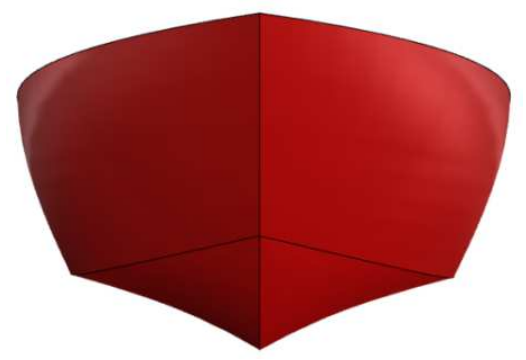

Fig. 1. Shape 1-3D Frontal View

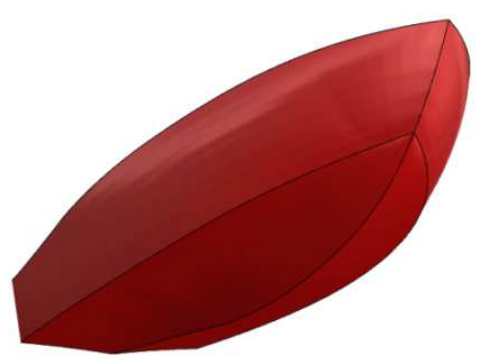

Fig. 2. Shape 1-3D Isometric View

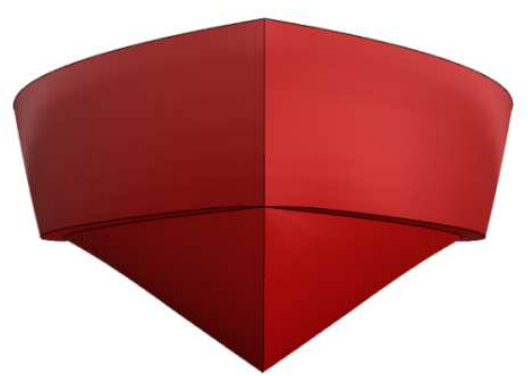

Fig. 3. Shape 2-3D Frontal View

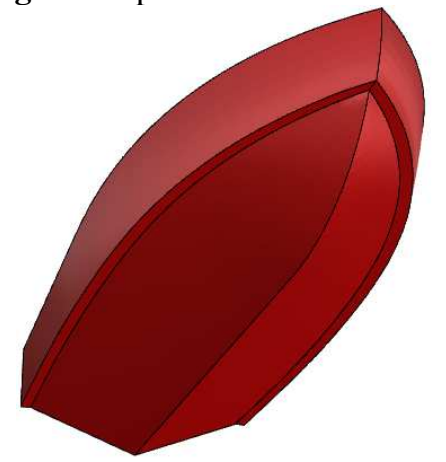

Fig. 4. Shape 2-3D Isometric View 
The main dimensions for the both shapes are presented in the table below. The second shape it is a little bit bigger because of redans.

Table 1. Main dimensions for the first form

\begin{tabular}{|l|c|c|}
\hline \multicolumn{3}{|c|}{ Main characteristics } \\
\hline & $\begin{array}{c}\text { Shape } \\
1\end{array}$ & $\begin{array}{c}\text { Shape } \\
2\end{array}$ \\
\hline Length over all, $L_{O A}[\mathrm{~m}]$ & 5.6 & 5.9 \\
\hline Length of waterline, $L_{W}[\mathrm{~m}]$ & 4.93 & 5.47 \\
\hline Beam, $B[\mathrm{~m}]$ & 1.9 & 2.3 \\
\hline Depth, $D[\mathrm{~m}]$ & 1.18 & 1.0 \\
\hline Draft, $T[\mathrm{~m}]$ & 0.39 & 0.41 \\
\hline $\begin{array}{l}\text { Longitudinal center of buoyancy, } \\
L C B[\mathrm{~m}]\end{array}$ & 2.36 & 2.43 \\
\hline Volumetric displacement, $\nabla\left[\mathrm{m}^{3}\right]$ & 1.91 & 1.86 \\
\hline Design speed, $v[\mathrm{~m} / \mathrm{s}]$ & 5 & 5 \\
\hline Froude number, $F_{n}$ & 1.78 & 1.77 \\
\hline
\end{tabular}

As mentioned above in this preliminary design stage the main dimensions will be adopted so that the ship is in transient regime. For this, the following condition must be fulfilled:

$$
\begin{aligned}
& 1<\mathrm{Fn}_{\bar{V}}<3 \\
& \mathrm{Fn}_{\mathrm{W}}=\frac{\mathrm{v}}{\sqrt{\mathrm{g}_{\mathrm{W}}^{15}}}=\frac{5}{\sqrt{9.81 \cdot 1.91^{1.3}}}=1.78
\end{aligned}
$$

The disadvantage of this type of bottom is given by the expensive manufacturing technology, but this is compensated by the fact that the boat will be made of aluminum, which is no longer an impediment, aluminum being a more malleable alloy. The solution to adopt a longitudinal redan was to improve the flow and avoid flushing the shells at the regime speed..

For the second shape the flow around the stern will be more smooth, due to the shape of the bow and the longitudinal redan. The longitudinal redan will have a significant part in the design of the boat.

Since CFD results obtained on the basis of the first shape at a speed of $5 \mathrm{~m} / \mathrm{s}$ were not favorable, the flow was very turbulent, and in the aft zone of the ship the water was climbing on the deck, we decided to genereate a new shape.

\section{THEORETICAL BACKGROUND}

Computational Fluid Dynamics (CFD) is the simulation of fluids using mathematical physical problem formulation and numerical methods the solve different problems.

The CFD methods are based on the incompressible fluid equations that are solved using Navier-Stokes (RANS) equations. In general, incompressible fluids are considered to be those fluids at which the Mach number is less than 0.3 . This hypothesis assumes that dynamic viscosity $\mu$ and density $\rho$ are constant and the Navier Stokes equation in vectorial form is written as:

$p\left(\frac{\partial v}{6 t}+v-\nabla v\right)=-\nabla p+\mu v^{2} v+1$

where:

- f represent other forces (gravity, centrifugal forces).

The explain of the Navier-Stokes system with the usual notation $\mathrm{u}, \mathrm{v}$ and $\mathrm{w}$ for the components of speed in the tree directions is:

$\rho\left(\frac{\partial u}{\partial t}+u \frac{\partial u}{\partial x}+v \frac{\partial u}{\partial y}+w \frac{\partial u}{\partial z}\right)=$ $-\frac{\partial v}{\partial x}+\mu\left(\frac{\partial^{2} u}{\partial x^{2}}+\frac{g^{2} u}{\partial y^{2}}+\frac{\partial^{2} u}{\partial z^{2}}\right)+\rho g x$

$\rho\left(\frac{\partial v}{\partial t}+u \frac{\partial v}{\partial x}+v \frac{\partial v}{\partial y}+w \frac{\partial v}{\partial z}\right)=$ $-\frac{\partial z}{\partial y}+\mu\left(\frac{g^{2} v}{\partial x^{2}}+\frac{\partial^{2} v}{\partial y^{2}}+\frac{g^{2} v}{\partial z^{2}}\right)+\rho g_{y}$

$\rho\left(\frac{\partial w}{\partial t}+u \frac{\partial w}{\partial x}+v \frac{\partial w}{\partial y}+w \frac{\partial w}{\partial z}\right)=$ $-\frac{\partial p}{\partial z}+\mu\left(\frac{\partial^{2} w}{\partial x^{2}}+\frac{g^{2} w}{\partial y^{2}}+\frac{\partial^{2} w}{\partial z^{2}}\right)+\rho g_{z}$

The continuity equation is:

$\frac{\partial p}{\partial t}+\frac{\partial(\rho u)}{\partial x}+\frac{\partial(\rho v)}{\partial y}+\frac{\partial(\rho w)}{\partial z}=0$

For incompressible fluids, the density being constant, the continuity equation is:

$\frac{\partial u}{\partial x}+\frac{\partial v}{\partial y}+\frac{\partial w}{\partial z}=0$. 


\section{MESH}

The mesh was calculated using the Courand number, which can be written as:

$$
C_{0}=\frac{\Delta t F_{c}}{V c}=\Delta t \sum_{f} \frac{\operatorname{Max}(-F(U), 0}{V c}
$$

where:

- $\Delta t$ is the time increment;

- Fc is the total flux of cells;

$-\mathrm{Vc}$ is the velocity.

Buffer Layer: $5<y+<30$. For this analyzes $y+$ has a value of 30 .

Dimensionless wall distance is calculated using the next formula:

$y^{+}=\frac{u_{z} \cdot y}{y}$

where:

- $\mathrm{u}_{\mathrm{t}}$ is the friction velocity;

- $y$ is the distance to the nearest wall;

$-\theta$ is the local kinematic viscosity.

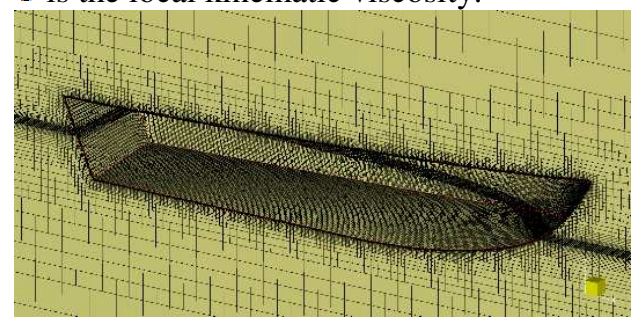

Fig. 5. Mesh for the first shape

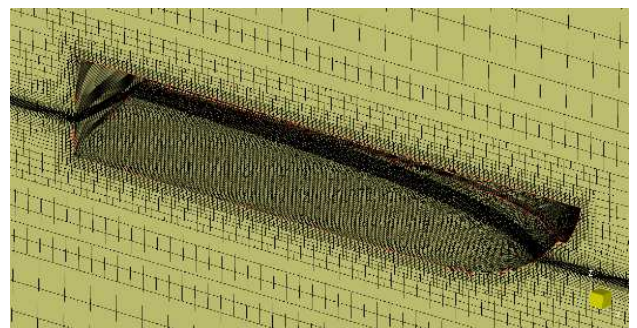

Fig. 6. Mesh for the second shape

\section{RESULTS}

For the first shape the results of the flow around the hull were not very satisfying. The disadvantages of this shape were:

- the waves generated at the aft part of the hull was very high, above the maximum depth, which means the water was getting in the boat;
- the flow around the fore part of the boat was very turbulent;

- the pressure on the bottom of the boat was very high, the aft part being submerged below the free surface of the water.

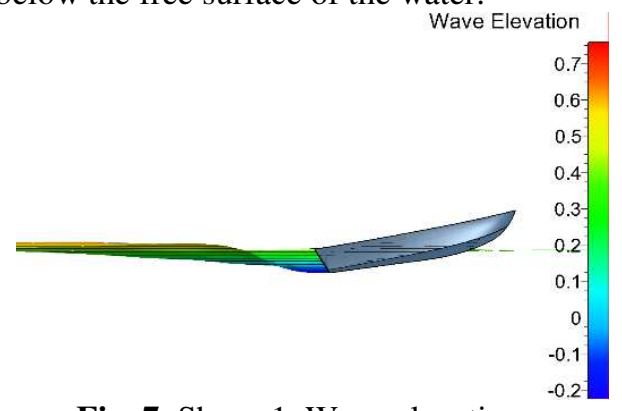

Fig. 7. Shape 1. Wave elevationLongitudinal view

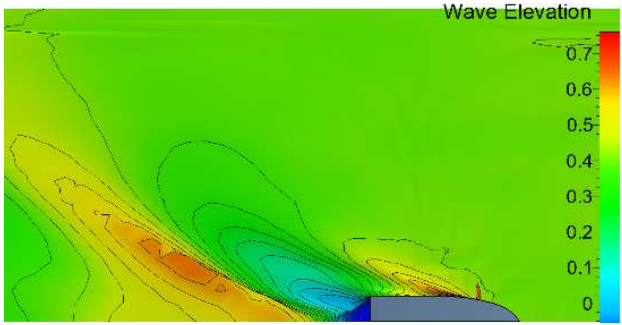

Fig. 8. Shape 1. Wave elevation Top view

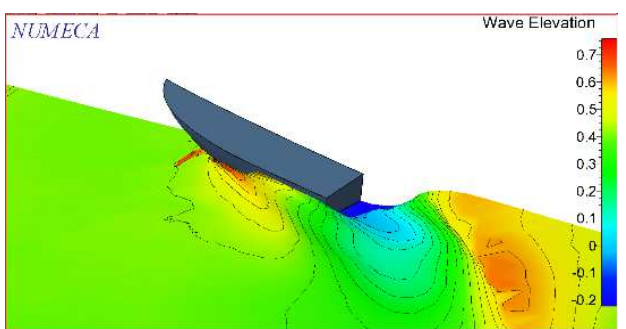

Fig. 9. Shape 1. Wave elevation Isometric view

The changes made for the second shape lead to significant improvments of the flow around the boat. The trim angle have now a lower value, and the flow at the aft and fore part of the ship is more stable then the previous shape. Probably the main reason of these changes due to the longitudinal redan, and the bottom shape. 
NUMECA

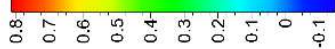

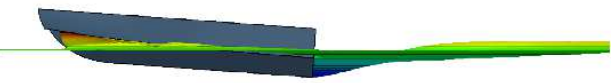

Fig. 10. Shape 2. Wave elevation Longitudinal view

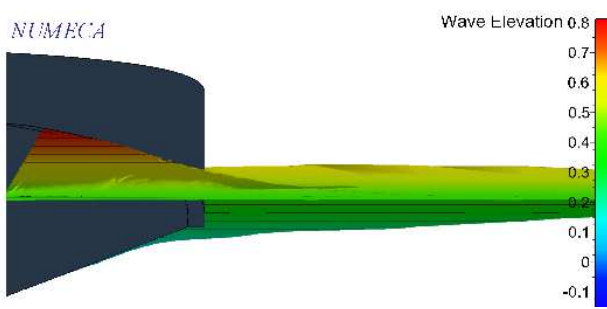

Fig. 11. Shape 2. Wave elevation Front view

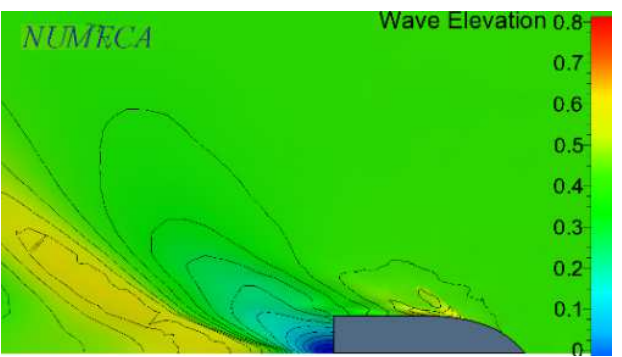

Fig. 12. Shape 2. Wave elevation - Top view

Both shapes were tested at a speed of 5 $\mathrm{m} / \mathrm{s}$. Total resitance of the ships is presented in tabel 3 .

Table 3. Total ship resistance

\begin{tabular}{|c|c|}
\hline Shape 1 & Shape 2 \\
\hline Rt $=1964[\mathrm{~N}]$ & $\mathbf{R t}=1204[\mathrm{~N}]$ \\
\hline
\end{tabular}

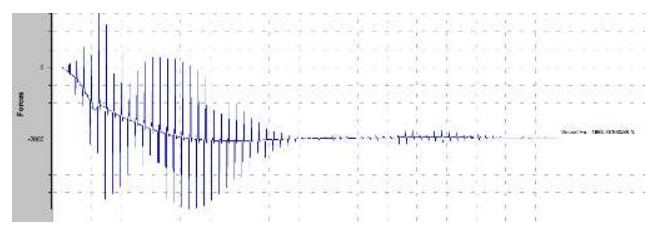

Fig. 13. Shape 1. Ship total resistance
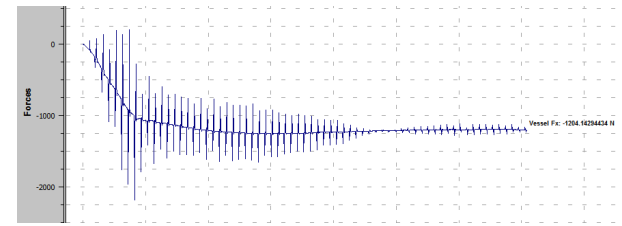

Fig. 14. Shape 2. Ship total resistance

\section{CONVERGENCE}

The convergence for each shape was obtained after 5000 iterative cycles. The residues for both shapes were graphically displayed, so any error of calculation could be identified in time.

\section{CONCLUSIONS}

In conclusion, it is clear that the second shape is better than the first one. That is because of the shapes of the second boat and the longitudinal redan.

The second shape has a lower resitance, a good flow around its hull and a better trim angle, compared with the first one. The diferrence on hull resistance between shape 1 and 2 is about $38 \%$. This means also the power of motor engine can decreased with $38 \%$.

Based on the results obtained so far, we are going to analyze the flow of the shape 2 in planning regime to see how the boat will behave at higher speeds.

After the ship form optimization is finished, a scale model will be made to see the exact phenomena that apper in a towing tank using experimental test and compare the results with the ones obtained with CFD methos.

The hull shell and the hull framework will be build entire of aluminum and not PFAS (Fibreglass Reinforced Polyester) because the total weight of the boat will be considerably reduced. 


\section{Acknowledgements}

The research was supported by the Naval Architecture Faculty, in "Dunarea de Jos" University of Galati, which is greatly acknowledged.

\section{REFERENCES}

[1]. Obreja, C.D., "Particularitatile hidrodinamice ale proiectarii navelor mici", Editura Fundatiei Universitare, Dunarea de Jos, Galati.

[2]. ISIS-CFD Centrale Nantes, CNRS

[3]. www.wikipedia.org

Paper received on December $31^{\text {th }}, 2018$ 